\title{
IL DIVIETO DELL'USURA NELLA SOCIETÀ CRISTIANA Valore morale, significato storico e implicazioni economiche
}

\author{
PAOLO ZANOTTO*
}

Resumen: El presente trabajo estudia las principales funciones, económicas y morales, de la prohibición del interés en el prestamo de dinero que se estableció, en época medieval, en la respublica christiana. Igualmente, se analizan los beneficios en términos sociales que esa norma produjo y las consecuencias negativas que, en cambio, se originaron en el trabajo del ser humano de su anulación por parte de los teóricos de la economía clásica en la época moderna.

Abstract: This work studies the main functions, economic and moral, of the prohibition of interest in loans of money which was settled down, at medieval time, in the christiana respublica. Also, it analyses the benefits in social terms that this norm produced and the negative consequences that, however, were originated in the human being's work of their cancellation on the part of the theorists of the classical economy at the modern time.

Palabras clave: divieto di usura; società cristiana; morale cattolica; vangelo; depositum irregulare; libertà d'intrapresa; capitalismo; alienazione.

Clasificación JEL: B11, B31, B53

$\left({ }^{*}\right)$ Dottore di ricerca in "Storia del pensiero politico europeo moderno e contemporaneo». Autore di un volume su Il movimento libertario americano dagli anni sessanta ad oggi: radici storico-dottrinali e discriminanti ideologico-politiche, Siena, Università degli Studi di Siena-Di. Gips (Collana «Monografie»), 2001, e di uno studio su Liberalismo e tradizione cattolica. Osservazioni critiche su Juan de Mariana (1535-1624), Roma-Soveria Mannelli (CZ), Istituto Acton-Rubbettino Editore (Quaderni dell'Istituto Acton, n. 16), 2004.

Procesos de Mercado: Revista Europea de Economía Política Vol. II, n. ${ }^{\circ}$ 2, Otoño 2005, pp. 81 a 118 


\section{ETICA \& MERCATO}

Lo storico delle idee e delle forme politiche, il filosofo della politica o lo storico del pensiero economico che decidessero di misurarsi con la dottrina libertarian avrebbero l'onere di chiarire, prima, che cosa intendano - di preciso - con tale termine. Al centro di numerose controversie infatti si pone, sovente, un fraintendimento lessicale. Molti studiosi individuano le lineeguida del pensiero libertario nell'opera di Murray N. Rothbard. Anche nella produzione di tale autore, tuttavia, è ravvisabile una chiara evoluzione, della quale è opportuno prendere atto, facendo tesoro della sua esperienza umana e del suo percorso intellettuale.

Il peculiare clima che caratterizzò gli anni sessanta e settanta del Novecento indusse Rothbard all'attivismo For a New Liberty, spingendolo a battersi in favore dell'economia di mercato dalla sponda della New Left. La prospettiva etica che, a quell'epoca, animava lo sparuto nucleo d'intellettuali libertarî è efficacemente sintetizzata in un libro che scrisse l'allora giovane economista Walter E. Block con l'intento dichiarato di Difendere l'indifendibile ${ }^{1}$. In tale esercizio di spregiudicata ars rhetorica si portavano alle loro inesorabili conseguenze le idee già espresse in proposito dal medico franco-olandese Bernard de Mandeville in un suo celebre apologo ${ }^{2}$, in cui si erano gettate le premesse per un peculiare état d'esprit, propedeutico ad un superamento dei residui di morale cristiana ancora presenti — a varî livelli-

\footnotetext{
1 Cfr. Block (1991), in cui si tenta di rivalutare la funzione sociale svolta da figure come la prostituta, il ruffiano, lo spacciatore di droga, il tossicodipendente, il ricattatore, il calunniatore, il diffamatore, il tassista abusivo, il bagarino, il poliziotto corrotto, il falsario ed altre ancora. La corretta prospettiva etica, opposta a quella sostenuta nel saggio di Block, è tradita dal provocatorio ossimoro contenuto nel titolo; prospettiva di cui, peraltro, lo stesso autore sostiene di essersi riappropriato in seguito: si veda in proposito il Mea culpa riportato nella 'postfazione' (247-248).

2 Cfr. Mandeville (1714) e (1724).
} 
nella società moderna e che sarebbe successivamente giunto a maturazione, culminando con una vera e propria Difesa dell'usura, ad opera di Jeremy Bentham ${ }^{3}$.

In seguito, tuttavia, Rothbard ha chiaramente indicato la via da seguire nel suo classico che, non a caso, s'intitola The Ethics of Liberty ${ }^{4}$. In quello che rappresenta il prodotto maturo della sua riflessione filosofico-politica, l'autore ha cercato di costruire una morale adeguata a sorreggere un sistema di libero mercato, inteso come sistema della libertà naturale dell'uomo. In sostanza, l'obiettivo che si è posto lo studioso americano ha coinciso con la fondazione razionale di un'etica positiva della libertà. Secondo Rothbard, l'economia è certamente in grado di contribuire, in una qualche misura, alla difesa della libertà individuale, e tuttavia essa si rivela incapace, da sola, di dare origine ad un'autentica filosofia politica tesa a tal fine. Per emettere giudizî politici si richiedono giudizî di valore; ragion per cui la filosofia politica è necessariamente etica e, pertanto, occorre progettare un sistema etico positivo al fine di poter difendere, con solide argomentazioni, la causa della libertà. Nella sua basilare History of Economic Thought, pubblicata postuma nel 1995, Rothbard ha infine esposto la storia del pensiero economico secondo una 'prospettiva austriaca', esprimendo così le fondamenta storico-dottrinarie della propria teoria ${ }^{5}$.

Ciò che emerge nettamente dall'analisi rothbardiana - peraltro incompiuta a causa della prematura scomparsa- è la necessità di un radicale ripensamento dei presupposti che si pongono alla base dell'economia di libero mercato. Ed è proprio tale rilettura l'elemento che maggiormente fatica a farsi strada presso alcuni dei suoi stessi estimatori. Non è infrequente, ad

\footnotetext{
${ }^{3}$ Cfr. Bentham (1787).

4 Cfr. Rothbard (1982).

5 Cfr. Rothbard (1995).
} 
esempio, imbattersi in sedicenti libertarians che tributano la loro ammirazione a supposti 'padri del liberalismo economico' come Adam Smith o David Ricardo. È opportuno rammentare, tuttavia, che tali autori rappresentano i più profondi negatori della teoria soggettivista e proto-austriaca - già formulata nell'ambito economico, sebbene incompiutamente, da pensatori di epoca basso-medioevale ed umanistico-rinascimentaleincarnando, di conseguenza, la più recisa negazione degli autentici valori libertarî.

\section{L'ECONOMIA È UNA SCIENZA 'AVALUTATIVA'?}

Lo stesso Joseph Alois Schumpeter, nella sua History of Economic Analysis ${ }^{6}$, pur richiamando la tradizionale alleanza della teoria economica con il liberalismo politico del secolo XIX, dichiarava esplicitamente che l'applicazione dell'analisi economica non poteva prescindere dai giudizî di valore etici, politici ed istituzionali. Egli sembrava addirittura voler andare oltre, affermando l'ineluttabile riferimento ai valori nella stessa fase analitica, oltre che nel momento applicativo. Il contributo specifico dello studioso austriaco, dunque, consisteva nel porsi l'interrogativo se fosse lecito per l'economista sottrarsi ad una presa di posizione in sede di valori nel momento dell'analisi scientifica, ossia prima ancora di passare all'applicazione pratica. La risposta che egli dava a tale problema era negativa, almeno per quanto concerneva quelle discipline aventi una diretta rilevanza per i rapporti umani, quale con tutta evidenza andava considerata quella riguardante il benessere materiale.

Ancor più saldamente che presso la cultura ellenica, le trattazioni economiche sviluppate all'interno della società

\footnotetext{
${ }^{6}$ Cfr. Schumpeter (1954).
} 
cristiana medioevale dai canonisti e dai filosofi scolastici si mostravano collegate alla dottrina dei doveri (etica) e alla dottrina della funzione della cosa pubblica (politica). Il punto di svolta era rappresentato da una nuova concezione del lavoro, non più inteso come un'attività essenzialmente negativa in quanto impedimento allo stato di contemplazione e di elevazione verso il divino, come avveniva presso i greci, bensì nobilitato in nome del comando di Dio. Per il cristianesimo il lavoro diveniva così un mezzo di elevazione oltre che di sostentamento, a patto che non fosse diretto esclusivamente al lucrum. Il lavoro non era percepito, pertanto, come fine a se stesso, ma si convertiva in valido strumento d'innalzamento morale.

Le tematiche predilette dagli scrittori dell'epoca furono, in special modo, quelle relative al 'giusto prezzo', al 'giusto salario' e all' usura'. Proprio la questione dell'usura avrebbe rappresentato, secondo Rothbard, 'la tragica falla' insita nella dottrina economica enucleata dai giuristi e dai teologi cattolici del medioevo ${ }^{7}$. A fronte del notevole impulso conferito al commercio con l'attenuazione delle restrizioni economiche, che avevano operato i canonisti e i romanisti dell'età media, non venne parallelamente estesa l'applicazione di tali dispositivi giuridico-morali alle severe proibizioni nei confronti del prestito ad interesse ${ }^{8}$. Rothbard è rimasto forse vittima qui —in un certo qual senso- di una visione falsata, nel tentativo di applicare di peso una concezione prettamente moderna ad una realtà storica affatto differente ${ }^{9}$. Egli ha dimostrato un'abilità certamente non comune nel saper leggere i fatti storici non con lo

7 «The usury prohibition was the tragic flaw in the economic views of medioeval jurists and theologians»: Rothbard (1995: I, 45).

8 Rothbard (1995: I, 42).

${ }^{9} \mathrm{Ha}$ forse finito per pesare eccessivamente, in tale frangente, la sua impostazione metodologica, di fedele economista misesiano, strettamente devoto all'astrattezza ed universalità delle leggi prasseologiche. 
sguardo velato dai pregiudizî moderni, bensì immedesimandosi nello spirito del tempo. E, tuttavia, è incorso in un comprensibile fraintendimento quando ha preteso di giudicare con gli occhî di un economista che viveva nella realtà -economicamente sviluppata e culturalmente secolarizzata- del XX secolo alcune misure specifiche che furono adottate in occasione del I Concilio di Nicea, tenutosi dal 19 giugno al 25 luglio del 325 dopo Cristo $^{10}$.

Chi scrive è pienamente consapevole di come il mettere in discussione la validità che il prestito ad interesse detiene per un'economia di mercato significhi scontrarsi con uno dei pochi tabù imposti dalle società liberali contemporanee. E tuttavia, una critica, che si spera di documentare appropriatamente, può forse riservare insospettati beneficî per il ripensamento globale —attualmente in atto- del sistema capitalistico. È esattamente questo lo spirito con cui sono state formulate le riflessioni che seguono.

\section{CAUSE ED EFFETTI DEL DIVIETO DELLE USURE}

Secondo una peculiare visione, che radicava nel contemptus mundi dell'esperienza monastica, «[p]er il vero santo non soltanto il prestito ad interessi, il piacere per la ricchezza o l'invidia dei poveri per essa, ma l'economia in genere è peccato» ${ }^{11}$. Mentre $1^{\prime}$ affare in senso proprio veniva detto commercium, infatti, il termine latino attraverso cui s'indicava ogni genere di attività economica era negotium, che —come si

${ }^{10}$ La condanna venne ribadita nei Concilî di Cartagine (345 d. C.), Aix (789), III Lateranense (1179), II di Lione (1274), Vienne (1311). La Chiesa continuò a condannare l'usura fino al 1745, quando papa Benedetto XIV riconobbe la liceità degli interessi nell'uso del denaro con l'enciclica Vix Pervenit.

11 Spengler (1923: 1351). 
poteva leggere nel Decretum Gratiani- «negat otium neque quaerit veram quietem, quae est Deus» ${ }^{12}$.

È certamente deprecabile la tendenza, «abbastanza diffusa nella storiografia giuridica ed economica, a ridurre la storia di quel divieto ad una sorta di epico certame tra una prassi negoziale impegnata solamente ad eludere il divieto stesso per insano amor del guadagno (aiutata nell'impresa da un nugolo di argomentatori prezzolati e sottili) e un'arcigna macchina inquisitoria dedita a scoprire - il più delle volte con successo - l'anfratto nel quale una mano scaltra aveva rimpiattato la clausola usuraria» ${ }^{13}$. È opportuno rilevare, infatti, che l'attenzione dei moralisti cattolici era stata carpita dal prestito ad interesse giacché in un'economia di sussistenza, quale è comunemente ritenuta quella dell'epoca alto-medioevale, non vi era posto per una funzione economica del credito nel senso moderno e, pertanto, i prestiti erano - solitamente- prestiti al consumo. Ciò che induceva a ricorrere ad un prestito era, quasi sempre, una condizione di emergenza che spingeva alla ricerca di liquidità nell'intento precipuo di far fronte alle spese correnti.

È dunque palese come, in tali circostanze, risultasse oltremodo facile per il prestatore trarre un vantaggio dallo stato di bisogno in cui versava il richiedente. Di qui la necessità morale di vigilare a tutela dei più deboli ${ }^{14}$. Sotto tale ispirazione sorsero i Monti di Pietà che emanciparono i poveri dall'oppressione usuraria concedendo prestiti su pegno senza interesse e restituendo al debitore, dietro il rimborso della somma prestata, $1^{\prime}$ oggetto pignorato ${ }^{15}$. In pratica, mentre il mutuatario non era materialmente in condizione di pagare un interesse, il mutuante

12 Citato in Spengler (1923: 1483, nota 10).

13 Santarelli (1989: 147-148). Per accedere a ricostruzioni storiche che non prestano il fianco a tali forzature polemiche, si consultino in particolare i lavori di Nelson (1967) e di Spicciani (1990).

14 Cfr. Vito (1967: 68).

15 Cfr. Vito (1967: 72). 
non aveva bisogno di ricevere un compenso per il prestito, cosicché - come ha opportunamente riconosciuto anche l'economista Francesco Vito- «[c]ol proibire l'usura per motivi religiosi la Chiesa rese un segnalato servizio alla società dell'alto Medio Evo» ${ }^{16}$. Non si può che concordare con Umberto Santarelli, infatti, quando sostiene che, da sempre, l'immagine di colui che s'approfitta dell'indigente, aggravando la sua già difficile situazione per trarne un utile a proprio esclusivo vantaggio, è stata universalmente interpretata come sintomo intollerabile di una situazione totalmente immorale ${ }^{17}$.

\section{RAGIONI ETICHE ED ECONOMICHE DELLA NORMA}

Era stata, di fatto, proprio l'esigenza pratica di salvaguardare le fasce sociali più disagiate a determinare l'adozione di misure precauzionali e non tanto un'aprioristica rigidità nell'esegesi biblica di alcuni passi contenuti nell'Antico e nel Nuovo Testamento. Il divieto biblico, tuttavia, esisteva, anche se non rappresentava affatto una singolare anomalia nel panorama delle antiche civiltà. Contrariamente a quanto affermato dallo stesso Rothbard infatti - che sosteneva la teoria secondo cui, con l'unica eccezione degli ebrei nei confronti degli altri ebrei, nel mondo antico nessuno, dalla Grecia alla Cina, dall'India alla Mesopotamia, aveva mai vietato il prestito ad interesse ${ }^{18}$ la proibizione dell'usura era un retaggio culturale tipico della tradizione occidentale, nel cui tronco si era innestato il

16 Ibidem.

17 Cfr. Santarelli (1989: 144).

18 «With one exception, no one in the ancient world - whether in Greece, China, India or Mesopotamia — prohibited interest. That exception was the Hebrews who, in an expression of narrow tribal morality, permitted charging interest to nonJews but prohibited it among Jews»: Rothbard (1995: I, 43, corsivo aggiunto). 
cristianesimo. È assai significativo — da questo punto di vistache, nei due volumi della sua pur ampia e pregevole Storia del pensiero economico, che va dall'antichità fino alla 'rivoluzione marginalista', Rothbard si sia soffermato a descrivere il sistema 'taoista', affermatosi in Cina nei cinque secoli precedenti alla venuta del Cristo (cap. I), per volgersi immediatamente dopo all'età media (cap. II), saltando così a pie' pari tutto il lungo periodo relativo alla storia romana.

Presso gli antichi romani, nondimeno, l'usura - nel senso di tutto quel denaro che era ottenuto senza lavoro, solo grazie allo scorrere del tempo - era detta fenus ${ }^{19}$ : dal tema $f$ - che si ritrova anche in femina, in cui era racchiuso il concetto di generazione (del denaro come dei figli). Nell'antica Roma esisteva un numero considerevole di leggi relative a tale problema. Già la legge delle XII tavole, del 451 a. C., proibiva chiaramente l'usura. Nel IV e III secolo a. C. si alternarono leggi che la proibivano ad altre che la permettevano, seppure a tassi controllati e relativamente bassi. Inoltre, similmente a quanto avveniva presso gli ebrei, anche qui erano essenzialmente i patrizî a praticarla ai danni dei plebei, ma non dei componenti della stessa gens aristocratica. Soltanto nei primi secoli della storia di Roma non era esistita alcuna regolamentazione rigida della questione: sia perché le leggi scritte si limitavano unicamente a poche sentenze, sia perché di per se stessa la si praticava poco, sia perché gli eventuali tassi usurarî erano fissati caso per caso nei singoli negozî giuridici d'indebitamento $^{20}$.

19 Derivato dalla voce verbale fenero, as, avi, atum, are (feneror, aris, atus sum, ari), 1. ${ }^{a}$ tr., che significava "prestare a frutto; dare ad interesse; speculare; render con usura»: Castiglioni-Mariotti (1966: 536).

$20 \mathrm{Su}$ tale problema nella Roma di età repubblicana e durante i primi trecento anni dell'Impero, si consulti lo studio di Andreau (1999). Si noti, in particolare, la distinzione - approfondita nei capp. II e III - tra coloro che prestavano denaro ad interesse (feneratores) e i banchieri di professione (argentarii), che svolgevano una funzione alquanto differente e che, comunque, comparvero solo - all'incirca- 
Non c'è alcun dubbio, nondimeno, che anche nel Vecchio Testamento l'argomento fosse piuttosto sentito: Rothbard accenna, ad esempio, al Salmo $14^{21}$. Nelle pagine vetero-testamentarie, tuttavia, è possibile rinvenire fonti ben più incisive. Le Sacre Scritture contenevano infatti un complesso normativo, di carattere giuridico-morale, nel quale si trovavano codificate le clausole del patto stipulato fra Jhwh ed il Popolo ricondotto dall'Egitto alla Terra promessa: si trattava del cosiddetto 'Codice dell'Alleanza'. Qui il divieto si trovava chiaramente e ripetutamente formulato ${ }^{22}$. L'espresso riferimento alla condizione d'indigenza del mutuatario si trova riportato - ad esempioin Exodus, a fianco della formale enunciazione del divieto: «Se avrai prestato del denaro a qualche povero del mio popolo che abita con te, non lo tormenterai come un esattore, né l'opprimerai con le usure. Se avrai ricevuto in pegno dal tuo prossimo il suo mantello, glielo renderai prima del tramonto del sole $\{S i$ autem argentum mutuum dederis fratri tuo paperi apud te, non eris eum urgens, non imponens ei usuram. Si autem pignus in pignum acceperis vestimentum proximi, ante solis occasum reddes ei\}; perché è quello il solo indumento col quale ei cuopre la carne sua, e non ne ha altro per dormirvi; se griderà a me, io l'esaudirò, perché sono misericordioso ${ }^{23}$. L'impianto della norma appare evidente: mutuatario è, per definizione, un povero, la cui situazione costituisce il presupposto di fatto tipico ed immancabile del mutuo; l'inevitabile conseguenza che da ciò

intorno al 310 avanti Cristo. Nel cap. VIII, espressamente dedicato al tasso d'interesse, si spiega inoltre il modo in cui si tentò frequentemente di limitare i prestiti fruttiferi attraverso la legislazione, come avvenne ad esempio con la lex Cornelia Pompeia dell'88 avanti Cristo. Sul tema si veda anche Andreau (1987).

21 «Signore, chi potrà albergare nella tua tenda? [...] Chi il suo danaro non dà ad usura, e doni non accetta contro l'innocente»: Sal 14, 1 e 5.

22 Cfr. Santarelli (1989: 144-145).

23 Es 22, 24-26. Il termine ebraico che il sacerdote Giovanni Giovannozzi - in Ricciotti (1940) - ha reso con 'esattore' è kenosheh, che letteralmente stava per 'creditore che presta ad interesse'; quello che ha reso con 'usure' è neshek, alla lettera 'morso'. 
deriva direttamente è il divieto assoluto di esercitare la pratica usuraria ed il significativo limite temporale legato all'eventuale pegno del mantello ${ }^{24}$. Per comprendere la ratio della norma è sufficiente far mente locale alla forte escursione termica che caratterizzava il clima delle regioni in cui, all'epoca, era stanziata la popolazione ebraica: il povero era in grado di privarsi del mantello durante le ore diurne, ma al calar della notte lo necessitava assolutamente ${ }^{25}$; tanto più che, se si deve credere al detto islamico, «l'abito preso a prestito non tiene caldo». È superfluo chiosare: cessante ratione legis, cessat ipsa lex.

Il secondo testo di riferimento sulla condanna dell'usura si trovava contenuto nel Deuteronomio: «Non presterai ad interesse ad un tuo fratello né denaro né alimenti né un'altra cosa; allo straniero sì. Al fratello tuo invece presterai senza interesse ciò di cui ha bisogno [...]» ${ }^{26}$. Sorvolando sul nuovo elemento sostanziale contenuto in questo secondo testo, rappresentato dalla differente regolamentazione del mutuo contratto nei confronti del 'fratello' rispetto a quello stipulato con lo 'straniero', sarà sufficiente concentrarsi, qui, sull'approfondimento dell'elemento formale. Si tratta della descrizione precisa di quei beni che possono costituire l'oggetto del mutuo di cui s'impone la gratuità. Essi consistono, essenzialmente, in quelli che 'romanisticamente' si è soliti definire 'di genere' o 'fungibili'. Di particolare interesse è la sottolineatura —nel testo deuteronomico-della presenza, fra questi, dei viveri: la loro dazione a mutuo, infatti, è un indiscutibile segnale della condizione di assoluta indigenza in cui doveva versare il mutuatario.

$V^{\prime}$ era inoltre un terzo ammonimento, contenuto nel Levitico: «Se un tuo fratello s'è impoverito, ed è caduto in basso stato,

\footnotetext{
24 Cfr. Santarelli (1989:145).

25 Ibidem.

26 «Non foeneraberis fratri tuo usuram argenti, et usuram ciborum, et usuram cuiuslibet rei quamcumque dederis mutuam. Alieno foeneraberis, fratri autem tuo non foeneraberis»: Dt 23, 19-20.
} 
e tu l'hai raccolto come un forestiero ed un ospite, e vive con te, non ricevere da lui usura, né più di quello che tu gli hai dato. Temi il tuo Dio, in modo che il tuo fratello possa vivere presso di te. Non gli prestare il tuo denaro ad usura, e dei generi datigli non esiger da lui il sovrappiù. Io il Signore Dio vostro, che vi cavai dall'Egitto, per darvi la terra di Canaan ed essere il vostro Dio» 27 . Tra i riferimenti di carattere puramente 'sapienziale' andrebbero inclusi, infine, altri passi vetero-testamentarîi ${ }^{28}$, fra cui il già citato Salmo 14.

\section{SAN LUCA: UNA PROSPETTIVA GIURIDICA O MORALE?}

Tutti i summenzionati precetti, d'altronde, appartenevano al corpus etico-giuridico contenuto nell'Antico Testamento. A quel complesso di norme, cioè, che disciplinavano la vita del popolo ebraico. Si è visto come, addirittura, alcuni di essi tendessero a distinguere l'atteggiamento da adottare se ci si fosse trovati al cospetto di un altro ebreo o, viceversa, se il rapporto avesse riguardato un ebreo ed un gentile ${ }^{29}$. Con la Cristianità medioevale si era, tuttavia, in presenza di una nuova alleanza ed il testo di riferimento era costituito dalla parola del Cristo. Era, dunque, presente nella produzione neo-testamentaria uno scritto che confermasse la sanzione biblica precedente? Il quesito trovò una soluzione affermativa, individuando la possibilità di un richiamo in un celebre passo del vangelo di Luca: «[...] e date in prestito senza speranza di ricambio \{et mutuum date nihil inde sperantes $\}{ }^{30}$. La richiesta, insieme esplicita ed estremamente

27 Lv 25, 35-38

28 Cfr. ad esempio Prv 28, 8; Ez 18, 8.13 e 17, nonché ivi, 22, 12.

${ }^{29}$ L'estensione del comandamento a tutti, stranieri compresi, nel cristianesimo primitivo è testimonianza della prospettiva autenticamente 'cattolica' del nuovo credo.

30 Lc 6, 35. 
esigente, sembrò non lasciare spazio alcuno alla libera interpretazione. Di conseguenza, per il cristiano il mutuo non poteva essere se non gratuito e l'usura, per tenue che fosse, andava irrimediabilmente condannata come illecita. Tale visione, tuttavia, appariva viziata da opposti fraintendimenti. La conseguente interpretazione risultava, pertanto, edulcorata ed eccessiva ad un tempo.

Edulcorata poiché, per la versione latina contenuta nella Vulgata, Girolamo - pur rimanendo rigorosamente fedele al testo greco- si avvalse in maniera ineccepibile del vocabolario tecnico-giuridico romano e rese, conseguentemente, l'espressione originale scrivendo тutuum date. A tal proposito, il Santarelli ha giustamente osservato come, «nel mutuo romano, [...] re contrahitur obligatio e non vi può perciò esser differenza quantitativa tra pecunia data (che fonda l'obligatio) e pecunia restituta (che l'obligatio estingue); sicché quel nihil che Girolamo scrisse non poteva in alcun modo riguardar l'usura, la cui corresponsione non poteva trovare il proprio titolo nel mutuum dare (ma, semmai, solo in un negozio aggiunto al mutuo anche se ad esso 'funzionale': cioè nella stipulatio usurarum). Se, dunque, con linguaggio giuridicamente così esatto, Girolamo scrisse che si doveva nihil sperare dalla mutui datio, quel nihil non poteva di certo riferirsi alle usurae, ma doveva per forza riguardare la stessa pecunia mutuata» ${ }^{31}$.

Estrapolare una frase da un contesto più ampio, d'altra parte, rischia sempre di falsarne il senso. Ciò è ancor più vero per un testo come il vangelo. Giova riportare, dunque, anche quanto l'evangelista premetteva al fine di motivare adeguatamente il comando: "Ciò che voi volete che gli uomini facciano a voi, fatelo voi pure a loro. Se voi amate coloro che vi amano, qual merito ne avete? Anche i peccatori amano coloro che li amano. E se fate del bene a coloro che lo fanno

31 Santarelli (1989: 153). 
a voi, qual merito ne avete? I peccatori fanno altrettanto. E se prestate denaro a coloro, dai quali sperate di ricavarne, qual merito ne avete? Anche i peccatori prestano ai peccatori, per ricevere altrettanto \{peccatores peccatoribus faenerantur ut recipiant aequalia $»^{32}$. È a questo punto che si trova inserito il monito: «Ma voi amate i vostri nemici; fate del bene e date in prestito senza speranza di ricambio»; Gesù prosegue assicurando come, in tal modo, "grande sarà la vostra ricompensa e voi sarete $i$ figli dell'Altissimo, ch'è buono pure verso gl'ingrati, e i cattivi. Siate dunque misericordiosi, com'è misericordioso il Padre vostro» ${ }^{33}$.

L'ammonimento di Gesù ci viene trasmesso dall'evangelista come una specificazione del comandamento generale di amare anche i proprî nemici, facendo loro del bene ugualmente a quanto si fa con gli amici. Chi abbia dimestichezza con 1'Antico Testamento, assicura il biblista Angelo Tosato, «non fa fatica a scorgere, sia nel comando più generale che in quello più specifico, un esplicito richiamo alla legge antica e insieme al suo superamento» ${ }^{34}$. Mosè, infatti, aveva comandato: «Amerai il tuo prossimo come te stesso»35. Il riferimento sembrerebbe rivolto, cioè, unicamente agli altri Israeliti ${ }^{36}$. L'ottica universale del cristianesimo avrebbe esteso la regola, ridefinendo il concetto stesso di 'prossimo'. Appare più che evidente a questo punto come, con quel nihil, non s'intendesse semplicemente spingere a rinunciare all'interesse maturato sulla somma prestata, bensì a predisporre l'animo al fine di poter dare a mutuo senza pretendere di ricevere nulla in cambio, neppure quella stessa somma mutuata (aequalia) che anche i peccatori esigevano di riottenere a tempo debito dai loro compari, secondo le regole

\footnotetext{
32 Lc 6, 31-34.

33 Lc 6, 35-36.

34 Tosato (2002: 273).

35 Lv 19, 18.

36 Cfr. Tosato (2002: 273).
} 
proprie del ius. In questo senso l'interpretazione risulta, come si diceva, edulcorata ${ }^{37}$.

Eppure la lettura di Luca fu, allo stesso tempo, decisamente eccessiva. È ormai chiaro, infatti, come ci si trovasse di fronte ad un semplice 'consiglio' e non ad un vero e proprio 'precetto' evangelico ${ }^{38}$. L'eccesso consistette dunque nel tentativo di omologare, in maniera assolutamente arbitraria, la regola evangelica alla normativa mosaica che vietava al pio israelita di reclamare usura dal proprio 'fratello'. In tal modo, infatti, si pretendeva di trasformare un'esortazione morale, prettamente interiore, in un canone sostanzialmente giuridico ${ }^{39}$. Gesù non aveva inteso affatto insinuare che il ricevere, o anche soltanto il pattuire, un giusto interesse fosse da considerarsi come un qualcosa di 'oggettivamente' illecito nella propria materialità di comportamento. Era ben altro che contava nella Sua prospettiva: si trattava di non giocare se stessi e la propria 'salvezza' nella vana e mondanissima speranza di ricevere qualcosa da uno qualsiasi di tali negozîi ${ }^{40}$.

Sotto il profilo degli avvenimenti storici conviene rammentare, peraltro, come la stessa proibizione di Nicea riguardasse unicamente la particolare categoria dei chierici ${ }^{41}$, venendo estesa anche ai laici solo in un secondo momento. L'ammonimento riportato dall'evangelista, inoltre, non riguardava affatto

37 Cfr. Santarelli (1989: 153-154).

38 Sulla distinzione fra 'precetti' e 'consigli' evangelici, quali espressioni della struttura della Legge cristiana, si veda Aquino (1266-73: I-II, q. 106, a. 1).

39 Cfr. Santarelli (1989: 154).

40 Ibidem.

41 XVII. Dei chierici che esercitano l'usura: «Poiché molti che sono soggetti ad una regola religiosa, trascinati da avarizia e da volgare desiderio di guadagno, e dimenticata la divina Scrittura, che dice: Non ha dato il suo denaro ad interesse (Sal $14,5)$, prestando, esigono un interesse, il santo e grande sinodo ha creduto giusto che se qualcuno, dopo la presente disposizione prenderà usura, o farà questo mestiere d'usuraio in qualsiasi altra maniera, o esigerà una volta e mezza tanto, o si darà, in breve, a qualche altro guadagno scandaloso, sarà radiato dal clero e considerato estraneo alla regola». 
l'applicazione di un equo interesse ai prestiti in denaro, bensì esortava il credente a non esitare nel concedere un prestito a chiunque ne avesse avuto bisogno, perfino quando non si poteva sperare alcunché in cambio: un notevole incentivo, dunque, all'attività economica (che si regge in larga misura sulla circolazione dei capitali), oltre che un atto di profonda carità cristiana nei confronti del prossimo. Tale interpretazione trova conforto nel corrispondente passo dell'apostolo Matteo, che volgeva il monito in positivo e non presentava l'equivoco riferimento all'interesse: «Da' a chi ti domanda, e non voltar le spalle a chi ti chiede un prestito» ${ }^{42}$. L'interpretazione restrittiva che voleva leggere nel passo evangelico un fermo divieto del prestito ad interesse, del resto, cozzava palesemente con la 'parabola dei talenti', dove il padrone rimproverava il proprio servo per non aver saputo mettere a frutto il denaro prestatogli, affidandolo alla cura dei banchieri in maniera tale da poterlo ritirare «con l'interesse $\{$ cum usura $\} »^{43}$.

\section{LA PROGRESSIVA STORICIZZAZIONE DEL DIVIETO}

La vera questione che la prassi e la riflessione teorica cristiana si trovarono, congiuntamente, a dover affrontare non fu tanto la ricerca di un meccanismo atto ad eludere il divieto biblico o, viceversa, di un dispositivo idoneo a perseguirne i contravventori, bensì - assai più in profondità- il problema consistette nell'individuare la stretta connessione che legava sapientemente tale divieto con una determinata struttura economico-sociale (di sussistenza). Una volta compreso tale aspetto, si trattava di prendere atto del passaggio che si era verificato verso un nuovo

$42 M t 5,42$.

${ }^{43}$ Mt 25, 26-27; similmente Lc 19, 23. 
assetto socio-economico, di tipo mercantile, constatando di conseguenza la perdita di significato di una conferma rigorosa e indiscriminata dell'antica proibizione nella nuova realtà ed elaborando, quindi, gli strumenti analitici che consentissero di distinguere le fattispecie in cui il divieto delle usure conservava intatto il proprio valore rispetto a quelle dove il mantenerlo aveva ormai perduto ogni significato ${ }^{44}$.

Il dibattito legato al divieto delle usure poteva ridursi, in buona sostanza, al problema di definire la natura e la funzione del denaro. Proprio l'impiego specifico che se ne fa ed il genere di utilità che ne traggono le parti contraenti, infatti, è in grado di determinare la diversa essenza che il denaro, di volta in volta, assume; essenza che incide direttamente, come ovvio, sul suo statuto. Ciò è, precisamente, quanto avvenne con il passaggio dall'età feudale, contrassegnata da un'economia di tipo 'curtense', all'età comunale, nel basso medioevo, contraddistinta invece dallo sviluppo di un'economia di mercato e dalla rapida ascesa del ceto mercantile ${ }^{45}$.

Tale passaggio - con tutto quanto esso implicò sotto il profilo economico, giuridico e morale - venne notevolmente agevolato dalla riflessione, sotto molti aspetti disincantata, che si sviluppò all'interno degli 'Ordini Mendicanti' e, soprattutto, in seno all'Ordine dei Frati Minori (Ordo Fratrum Minorum) fondato nel 1209 da Francesco d'Assisi. Il religioso provenzale Pierre de Jean Olivi ed il senese Bernardino degli Albizzeschi, entrambi francescani, così come l'allievo di quest'ultimo, il domenicano fiorentino Antonino Pierozzi —in particolare- si distinsero nel teorizzare il ruolo centrale che la capacità imprenditoriale e creativa dell'essere umano aveva quale stimolo per l'economia di mercato. Quanto a Francesco, che era figlio di un ricco mercante, aveva deciso di discostarsi radicalmente dal modello

${ }^{44}$ Cfr. Santarelli (1989: 148).

45 Cfr. Santarelli (1989: 149). 
di vita paterno in un émpito di emancipazione interiore. E, tuttavia, né lui né gli altri frati francescani equivocarono mai la povertà -che praticarono volontariamente e con intransigenza come virtù spirituale- con un disprezzo manicheo nei confronti della società mercantile, che conoscevano assai bene, o degli strumenti economici su cui tale realtà si fondava ${ }^{46}$.

La progressiva 'storicizzazione' del divieto, cominciata in epoca medioevale sotto l'impulso di alcuni esponenti del primo Ordine francescano, trovò un'emblematico sviluppo nella prima, durissima, polemica che Lutero condusse, da posizioni di fondamentalismo 'evangelico', contro le 'estorsioni usurarie' che - a suo dire- praticava la Chiesa di Roma ${ }^{47}$. Da parte sua Calvino, l'altro grande riformatore, rivendicò la reciproca autonomia di coscienza e norma biblica, liberando in tal modo l'azione pratica dalle opprimenti discipline che egli riteneva la ingabbiassero ${ }^{48}$. Affatto diversa si rivelò, per contro, la via che, in campo cattolico, perseguirono i teologi-giuristi della Seconda Scolastica ${ }^{49}$. Costoro, decisero di non avventurarsi in terreni piuttosto scoscesi ed insidiosi per il cattolico che intendeva rimanere fedele alla tradizione, come sarebbe stato l'operare dei semplici distinguo nel sistema normativo che disciplinava l'usura. Essi si rifiutarono, inoltre, di recidere il legame - altrettanto tradizionale - che manteneva unita la coscienza alla legge, come prevedeva uno dei più specifici postulati della Protesta. Di conseguenza, optarono per incentrare la propria riflessione sul più affidabile registro della 'natura delle cose', riconoscendo che l'essenza medesima del denaro era soggetta a mutazione in base all'impiego cui esso veniva, volta per volta, destinato. Da tale cambiamento, che andava inteso come 'oggettivo' nonostante derivasse da una causa soggettiva quale era l'uso che

\footnotetext{
46 Cfr. Santarelli (1989: 150).

47 Cfr. Nelson (1967: 55 ss.).

48 Nelson (1967: 107 ss.).

49 Cfr. Ambrosetti (1973: 23 ss.).
} 
se ne faceva, poteva e doveva scaturire un nuovo atteggiamento verso le regole di comportamento in relazione alla moneta ${ }^{50}$.

\section{IL DEPOSITO IRREGOLARE DI DENARO}

Non si deve dimenticare, inoltre, che il divieto rinveniva una solida giustificazione nell'inscindibilità dell'uso dalla proprietà nei già rammentati beni fungibili (qui primo usu consumuntur), di cui la moneta costituisce l'esempio per antonomasia. In base a tale visione, il mutuante non poteva pretendere, oltre alla restituzione del capitale dato in prestito, un compenso per l'uso di quello, giacché tale uso, risultando di fatto inscindibile dalla moneta, spettava al mutuatario, che ne diveniva in quel lasso di tempo proprietario a tutti gli effetti ${ }^{51}$. Precisamente su tali precetti giuridici s'imperniava anche la critica di Luis Saravia de la Calle e di altri scolastici spagnoli all'esercizio della banca con 'riserva frazionaria'52. Secondo detti autori, infatti, l'utilizzo a proprio beneficio, mediante la concessione di prestiti a terzi, del denaro depositato 'a vista' era da ritenersi illegittimo e supponeva un grave peccato ${ }^{53}$. Tale dottrina, peraltro, coincideva pienamente con quella già stabilita dagli autori classici del diritto romano, che a sua volta scaturiva naturalmente dall'essenza, causa e natura giuridica del contratto di 'deposito irregolare' (depositum irregulare) di denaro ${ }^{54}$.

\footnotetext{
50 Cfr. Santarelli (1989: 151-152).

51 Assai diverso era, invece, il caso in cui l'uso fosse distinto dalla cosa stessa (come ad esempio per la casa d'abitazione) dove si poteva ben esigere, oltre alla restituzione della cosa, anche una giusta rimunerazione per l'uso (il canone d'affitto).

52 Per una prospettiva critica sul sistema bancario con 'riserva frazionaria', si consulti Huerta de Soto (1998).

${ }^{53}$ Cfr. Huerta de Soto (2001: 57; trad. it. 70-71).

${ }^{54}$ Sulla singolare figura contrattuale del deposito irregolare, si può consultare Santarelli (1984: 67 ss.).
} 
Il deposito irregolare di denaro fu uno dei due tipi contrattuali che i giuristi medioevali idearono per rispondere all'esigenza di armonizzare i bisogni della società mercantile con le obbligazioni etico-religiose emanate dalla Chiesa. Al soddisfacimento del medesimo intento rispose anche, da un'altra prospettiva, la 'commenda'. Lo scopo sostanziale del contratto di deposito irregolare consistette nel mettere capitali a disposizione dell'attività mercantile. Attraverso tale istituto, in buona sostanza, si disciplinò l'affidamento ad un mercante (o ad un vero e proprio mediatore professionale nel traffico dei capitali, come erano i banchieri) d'una somma di denaro destinata a venire poi investita in affari di mercatura per esser quindi restituita al depositante assieme ad un interesse maturato sulla stessa. Tale interesse altro non rappresentava, in termini economici, se non una partecipazione al profitto d'impresa da parte di chi - direttamente (come nel caso che il deposito irregolare avvenisse nei confronti dello stesso mercante) o indirettamente (se sussisteva un'intermediazione bancaria) — aveva messo a disposizione il capitale ${ }^{55}$.

Ma la qualificazione come società del finanziamento dell'impresa altrui, con la conseguente affrancazione dal divieto del prestito ad interesse - che si rese possibile tramite la commenda e il depositum irregulare - fu l'esito di un processo graduale. Ciò che interessa notare qui è come l'istanza della prassi sia stata raccolta, in un primo momento, non dalla scienza giuridica ma dalla teologia. Come ha sottolineato il Santarelli: «rapportando i fatti nella loro immediatezza al precetto 'divino' della essenziale gratuità del mutuo fu possibile scoprire perché il finanziamento della mercatura dovesse porsi al di fuori dell'ambito di applicazione del divieto ${ }^{56}$. Nella Somma teologica di Tommaso d'Aquino il ragionamento economico è già

55 Cfr. Santarelli (1989: 164-165).

56 Santarelli (1989: 166). 
compiutamente svolto e le sue conseguenze risultano, ormai, ampiamente acquisite ${ }^{57}$. È così che, mentre discettava de peccato usurae, dopo essersi dedicato ad illustrare diffusamente l'effettiva illiceità dell'usura (accipere usuram pro pecunia mutuata est secundum se iniustum), l'Aquinate passava a domandarsi se non fosse stato tuttavia lecito il pretendere, in cambio del mutuo di denaro, qualche altro utile che non avesse coinciso con l'usura (utrum aliquis possit pro pecunia mutuata aliquam aliam commoditatem expetere $)^{58}$. Fra le possibili commoditates giudicate come accettabili rientrò, appunto, il finanziamento della mercatura.

\section{UN INSOSTITUIBILE IMPULSO PER LA LIBERA IMPRESA}

Ecco così che, nella condivisibile interpretazione del Sombart, anche le opinioni sul divieto del prestito ad interesse formulate dagli scolastici - e, in particolar modo, da quelli tardomedioevali- non rappresentarono affatto un ostacolo per lo sviluppo del capitalismo, bensì dovevano piuttosto contribuire a rinvigorirlo e a promuoverlo in misura essenziale ${ }^{59}$. Proprio la 'simpatia cordiale' e la 'piena comprensione' per lo 'slancio' preso dalla vita economica in quel tempo nel proprio paese, infatti, avrebbe indotto i tardi scolastici italiani a mantenersi aggrappati, con tanta tenacia, alla dottrina ecclesiastica dell'usu$\mathrm{ra}^{60}$. Il divieto del prestito ad interesse sulle labbra dei moralisti cattolici del XV e XVI secolo, insomma, stava a significare, in termini tecnici, che non si doveva impedire al denaro di mutarsi in capitale ${ }^{61}$.

57 Cfr. Santarelli (1989: 167).

58 Cfr. Aquino (1266-73: II-II, q. 78, a. 2).

59 Cfr. Sombart (1913: 194-195).

60 Cfr. Sombart (1913: 197).

61 Il concetto secondo cui tale divieto costituiva il più energico stimolo allo sviluppo del sistema capitalista, per quanto potesse apparire paradossale a prima 
In san Tommaso il concetto di 'capitale' era ancora in statu nascendi $\mathrm{e}$, tuttavia, egli già distingueva, come si è visto, sia pure unicamente per segni formali, il mero prestito di denaro dall'investimento di capitale. Cosicché, mentre non ammetteva il guadagno che derivava dal primo, riteneva del tutto legittimo quello scaturito dal secondo ${ }^{62}$. Con Bernardino ed Antonino, viceversa, 1 'idea si trova ormai sviluppata in perfetta chiarezza, tanto che lo stesso termine 'capitale' ha già un impiego corrente. Il futuro santo senese distingueva, ad esempio, fra mutuum usurarium e ratione capitalis ${ }^{63}$. Mentre secondo il suo discepolo fiorentino il capitale investito (ratio capitalis) ed il prestito di danaro (ratio mutui) andavano messi in reciproca, netta e sostanziale opposizione ${ }^{64}$. Nella forma del mutuo il denaro risultava sterile, non fruttando nulla, mentre sotto forma di capitale esso si mostrava fecondo giacché, come tale, possedeva una peculiare 'qualità creativa'65.

vista, studiando le fonti «appena quanto basta», s'imponeva per il Sombart «in modo tale che francamente non capisco bene come sino a ora nessuno abbia colto il nesso che li unisce. Forse dipende dalla circostanza che le fonti vennero per lo più sfruttate da profani di economia politica, privi di quella disciplina concettuale che dovremmo ammirare in Antonino e in Bernardino»: Sombart (1913: 198).

62 «Dicendum quod ille qui mutuat pecuniam transfert dominium pecuniae in eum qui mutuat. Unde ille cui pecunia mutuatur sub suo periculo tenet eam, et tenetur integre restituere. Unde non debet amplius exigere ille qui mutuavit. Sed ille qui committit pecuniam suam vel mercatori vel artifici per modum societatis cuiusdam, non transfert dominium pecuniae suae in illum, sed remanet eius, ita quod cum periculo ipsius mercator de ea negotiatur vel artifex operatur. Et ideo licite potest partem lucri inde provenientis expetere, tanquam de re sua»: Aquino (1266-73: II-II, q. 78, a. 2).

63 Cfr. Albizzeschi (1963: XLII, c. II). Sui temi trattati in queste pagine san Bernardino s'intrattenne anche in alcune delle sue celebri 'prediche volgari', pronunciate in Firenze e Siena fra il 1424 ed il 1427.

64 «Si [pecunia est tradita] per modum capitalis, seu rectae societatis, tunc in pacto esset, quod deberet eam solis mercatoribus fideliter deputare. Et haec ultima ratio videtur fortiter probare, quod non sit tradita dicta pecunia, nisi ut mutui rationem habens in quo spes lucri reprobatur»: Pierozzi (1440-59: II, 1, 6, par. 16).

65 «Illud quod in firmo proposito Domini sui est ordinatum ad aliquod probabile lucrum, non solum habet rationem simplicis pecuniae, sive rei, sed etiam ultra hoc 
In pratica, l'utile sul capitale non era affatto proibito, bensì perfettamente lecito; sia nel caso che derivasse da affari commerciali, sia che rappresentasse il frutto di un'impresa capitalistica, al pari che provenisse da un'assicurazione sui trasporti o dalla partecipazione ad un'impresa altrui ${ }^{66}$. S'imponeva un'unica limitazione: che il capitalista prendesse parte all'impresa direttamente, sia col guadagno che con le perdite eventuali. È per tale ragione che l'usura, dove si concedeva una somma in prestito ad un interesse fisso senza alcun rischio, veniva considerata illecita. Come ha giustamente osservato, in proposito, il Sombart: "Ciò dimostra con lampante chiarezza che l'opposizione tentata da qualcuno fra credito produttivo e credito consuntivo [con interesse permesso nel primo caso e vietato nel secondo] non rende pienamente giustizia alla dottrina scolastica del guadagno» ${ }^{67}$.

\section{DUE MODELLI A CONFRONTO}

Si tenterà adesso di esporre, in sintesi, quali siano le opposte logiche che marcano la differenza fra il modello 'cattolico' e quello 'protestante', relativamente all'aspetto economico della società; logiche che rendono apparentemente simili ma, in definitiva, non del tutto assimilabili fra loro i due modelli. Per far questo dovremo prima interrogarci su quali siano stati gli elementi caratteristici di entrambi. In cosa si differenzino, cioè, l'economia di mercato medioevale e quella moderna. Molte volte - e con esiti contrastanti- ci si è chiesti se le radici del

quamdam seminalem ratione lucrosi, quam communiter capitale vocamus. Ideo non solum reddi habet simplex valor ipsius, sed etiam valor superadjunctus»: Albizzeschi (1963: XXXIV, c. III).

${ }^{66}$ Cfr. Pierozzi (1440-59: II, 1, 6, parr. 15 e 29; nonché Albizzeschi (1963: XLII, cc. II e III).

67 Sombart (1913: 199). 
capitalismo vadano rintracciate nell'etica protestante, così come suggerì Max Weber, o piuttosto nella riflessione maturata in seno alla filosofia scolastica. Un primo equivoco sta certamente nel confondere lo 'spirito del capitalismo', di cui ha parlato Weber, con il capitalismo in quanto tale. Ma la questione sorge anche - e soprattutto - dalla fumosità della terminologia adottata: 'capitalismo' è, in effetti, una definizione che risente di un'antica connotazione polemica. Il termine venne infatti coniato ed utilizzato, in un primo momento, nell'ambito della cultura marxista (sebbene Marx, praticamente, non lo abbia usato) con l'intento di denunciare e criticare un sistema nel quale i lavoratori, che producevano la ricchezza del paese, non potevano poi goderne i frutti ${ }^{68}$.

Una volta introdotto il termine in ambito 'scientifico', se ne sono date molteplici definizioni non sempre sovrapponibili l'una all'altra né facilmente conciliabili fra loro. In esse ricorrono, tuttavia, alcuni elementi comuni che si possono estrapolare, nel tentativo di offrire una visione d'insieme: 1 ) l'economia capitalista è fondata, come il nome stesso rivela, sul capitale - che serve a finanziare l'impresa - e sullo scambio. Questi due fattori abbisognano, ovviamente, di un elemento fondamentale che è la moneta. Diversamente dal periodo feudale, che fu un"'economia naturale', in cui a) gli scambi erano scarsi e predominava il consumo diretto dei beni prodotti (autosussistenza); $b$ ) gli scambî avvenivano, comunque, sotto forma di baratto; 2) si ha una prevalenza del settore, cosiddetto, 'secondario' o industriale, rispetto a quello 'primario' o agricolo; 3) il motore che permette alla macchina di funzionare è una figura imprenditoriale intesa come homo oeconomicus, "al quale l'economia vale non come un ordine di mezzi ma come un ordine di fini tanto da costituire il dominio al quale va a consacrare le principali sue

\footnotetext{
68 Cfr. Finzi (1987: vol. I, 78).
} 
attività»69; una sorta di 'massimizzatore', cioè, che ha come obiettivo primario l'arricchimento personale ma come fine ultimo il successo della propria impresa, di cui mira ad ampliare indefinitamente la capacità produttiva, in quanto non considera il guadagno come un semplice mezzo per soddisfare alle proprie necessità materiali, bensì come il vero strumento per raggiungere lo scopo della sua vita che consiste nel potere economico fine a se stesso; 4) a fronte dell'imprenditore così inteso, nel moderno sistema economico capitalistico si ha il lavoro salariato: l'operaio non possiede i mezzi di produzione, che appartengono al datore di lavoro. Per vivere, di conseguenza, egli è costretto a 'vendere' la propria forzalavoro. In ciò si ha una differenza importante con le economie precedenti dove: a) la persona, e non la capacità di lavorare, era considerata una merce di scambio, come avveniva nei sistemi a produzione schiavistica, o $b$ ) il lavoratore, per quanto subordinato, possedeva i proprî 'ferri del mestiere', come succedeva sia nel sistema feudale che in quello artigianale di età comunale; 5) a partire dal XIX secolo, in particolare, si è avuta una progressiva urbanizzazione, evento indispensabile per l'assetto economico moderno. Nel mondo scaturito dalla 'rivoluzione commerciale' avvenuta nel basso medioevo, per contro, la città rimaneva comunque una realtà numericamente marginale e, in ogni caso, vincolata ad un'economia ancora sostanzialmente agraria; 6) nell'era industriale e postindustriale si è raggiunto il massimo sviluppo nella divisione del lavoro. Tale fenomeno si era già affacciato durante il periodo della 'rivoluzione commerciale', ma in quel tempo continuava a prevalere una divisione 'professionale' del lavoro; con l'avvento della 'rivoluzione industriale', invece, è subentrata una divisione 'tecnica'. Nel primo caso il lavoratore si specializzava in un dato mestiere, che corrispondeva ad una

\footnotetext{
69 Evola (1953: 114).
} 
precisa fase del processo produttivo (pettinatura, tessitura, tintoria, etc.); nel secondo egli si sarebbe dedicato ad un'unica operazione interna ad ogni fase della produzione (catena di montaggio $)^{70}$.

\section{VERSO UN'ALIENAZIONE DEL LAVORATORE?}

Ha ben messo in luce lo storico economico Carlo Maria Cipolla come, «[t]ra il 1780 e il 1850, in meno di tre generazioni, una profonda Rivoluzione che non aveva precedenti nella storia dell'umanità cambiò il volto dell'Inghilterra. Da allora il mondo non fu più lo stesso»71. D'altra parte, «[p]er le sfere scientifiche il divorzio effettivo dalla totalità significa la morte», ha acutamente posto in evidenza il filosofo esistenzialista Karl Jaspers $^{72}$, sottolineando inoltre come, conseguentemente alla 'rivoluzione scientifica', "[a]lla conoscenza sottentrano una tecnica e un mestiere meccanizzati, e alla cultura dello spirito, il cui indirizzo conoscitivo è sempre universale, anche nello studio di un settore specifico, si sostituiscono il possesso e l'esercizio di strumenti a cui non servirà essere perfetti» ${ }^{73}$.

A tale proposito, preme attirare l'attenzione su di un aspetto in particolare: si è detto di come, già nel borgo medioevale, molti fossero i lavoratori che compivano la propria opera alle dipendenze altrui. Lavoro subordinato e lavoro salariato, tuttavia, non rappresentano affatto la medesima cosa. L'artigiano che lavorava alle dipendenze del lanaiolo, ad esempio, era proprietario dei suoi mezzi di produzione (il telaio) ed instaurava quindi con il mercante-imprenditore un contratto

\footnotetext{
70 Cfr. Finzi (1987: 79-81).

71 Cipolla (1974: 411).

72 Jaspers (1925: 11).

73 Ibidem.
} 
sostanzialmente differente da quello che un operaio salariato sarebbe stato, poi, costretto a sottoscrivere con il proprio datore di lavoro, per il quale andava a compiere un servizio completamente diverso. Fra le differenze più evidenti e gravide d'implicazioni occorre tenere presente che l'artigiano era virtualmente in grado di collaborare con più imprenditori contemporaneamente, era libero di organizzare il suo lavoro come meglio credeva e poteva sempre cercare di emanciparsi mettendosi in proprio $^{74}$.

Ciò deriva dal fatto che l'articolazione fra arti e mestieri e, di conseguenza, la netta distinzione fra 'artista' ed 'artigiano' è - come la comune radice segnala - un fenomeno tipicamente moderno. Presso gli antichi, infatti, l'artifex coincideva, indifferentemente, con colui il quale esercitava un'arte o un mestiere $^{75}$, tanto che - in maniera rivelatrice- le due professioni erano ricomprese, nella società medioevale, sotto un'unica corporazione. Riguardo, poi, alla questione della divisione 'tecnica' del lavoro, che come ricordato ha preso piede in epoca moderna, merita riportare la concezione tradizionale, secondo cui gl'individui non sono oggetti intercambiabili -come vorrebbe il pensiero egualitario- bensì soggetti unici in sé. Ciascuno dovrebbe, in armonia con tale visione, poter svolgere una determinata funzione a cui la sua stessa natura, con le specifiche attitudini che questa implica, lo ha destinato: si noti, a tal proposito, come il termine 'mestiere' significhi propriamente 'funzione', secondo la sua derivazione etimologica dal latino ministerium ${ }^{76}$.

Lo svolgere una funzione differente da quella per cui saremmo naturalmente predisposti non può rappresentare, nella visione tradizionale, se non un grave turbamento che avrà una

\footnotetext{
74 Cfr. Finzi (1987: 81)

75 Cfr. Guénon (1945: 59).

76 Cfr. Guénon (1945: 61).
} 
ripercussione su tutta l'organizzazione sociale di cui ciascuno fa parte. Questo perché, secondo tale concezione, sono le qualità essenziali degli esseri umani a determinare la loro attività. Nella concezione moderna, all'opposto, tali qualità non contano e gl'individui vengono considerati come mere 'unità' intercambiabili e puramente numeriche. È così che ha origine la 'spersonalizzazione' dell'uomo, dato che quest'ottica non può logicamente condurre a null'altro se non all'esercizio di un'attività prettamente 'meccanica', nella quale finisce per non sussistere più alcunché di veramente umano in senso proprio $^{77}$.

Prova ne sia come, nel lavoro industriale, l'operaio non metta nulla di se stesso in quanto sta facendo; cosa che, peraltro, si avrebbe buona cura d'impedirgli qualora ne mostrasse la minima velleità. Preoccupazione che non sussiste, d'altronde, in quanto tutta la sua attività si riduce nel far funzionare una macchina. Egli, inoltre, è reso assolutamente privo d'iniziativa dalla 'formazione' professionale, a cui è sottoposto, la quale si rivela come l'antitesi dell'antico 'apprendistato', dato che egli non è più spinto ad affinare un'abilità personale ma viene indotto ad eseguire determinati movimenti 'meccanicamente' e sempre alla stessa maniera, senza che debba comprenderne la ragione ultima o preoccuparsi del risultato finale, giacché in realtà non è lui l'artefice del prodotto finito, bensì la macchina ${ }^{78}$. Anche il problema dei salarî è molto secondario, secondo quanto ha opportunamente rilevato il filosofo francese Gustave Thibon, poiché un «artigiano di paese che costruisce oggetti completi e tratta con una clientela viva è infinitamente più felice e soddisfatto dell'operaio d'officina, pur con uno standard di vita ben inferiore a quello di quest'ultimo» ${ }^{79}$.

\footnotetext{
77 Ibidem.

78 Cfr. Guénon (1945: 63).

79 Thibon (1940: 29, corsivo originale).
} 
In ciò, presumibilmente, consistette l'individuazione del capitalismo industriale - ai suoi esordî - come un sistema dominato da 'macchinarî satanici'80. E di fronte all'obiezione secondo cui la tecnica avrebbe sempre affiancato l'opera dell'uomo, giova rammentare l'opposta prospettiva che con la modernità si è affermata. Si può infatti sostenere che, per certi versi, la macchina appare come l'esatto opposto del primitivo utensile. Lungi dall'esserne un lineare perfezionamento, come alcuni pensano, essa obbedirebbe invece ad un principio diametralmente opposto. L'utensile costituiva infatti, in un certo qual modo, un 'prolungamento' dell'uomo stesso, consentendogli di meglio esprimere le proprie doti. La macchina, per contro, avrebbe convertito l'uomo in un suo anonimo elemento. Ridotto a servitore del macchinario, l'uomo avrebbe così finito per mutarsi egli stesso in ingranaggio, 'alienandosi' infine dalla propria natura ${ }^{81}$.

\section{CONSIDERAZIONI CONCLUSIVE}

Lo spunto di riflessione iniziale ha condotto lontano e qualche parola va, forse, spesa a chiarire il senso e la portata di talune affermazioni. Innanzi tutto, è opportuno specificare che considerare 'alienante' un'operazione, non implica necessariamente il ritenerla anche faticosa. I due concetti sono ben distinti fra loro e, molte volte, persino antitetici. Trascorrere ore ed ore di fronte ad un videogioco - ad esempio- è senza alcun dubbio alienante, per quanto certamente provochi un affaticamento fisico alquanto ridotto. Dedicarsi anima e corpo a coltivare duramente la terra, per contro, può essere un'opera sfiancante e, tuttavia, non produce alcun effetto alienante nell'individuo.

${ }^{80}$ Cfr. Polanyi (1944: 43).

81 Cfr. Guénon (1945: 63, nota 1). 
Ha scritto sempre Thibon che «[e]siste una vita dura e difficile che è umana: quella del contadino, del pastore, del soldato, del vecchio artigiano di paese...; esiste anche una vita molle e facile che è inumana e che genera la corruzione, la tristezza e l'eterna ribellione dell'essere che non svolge alcun ruolo vivente nella Città: quella per esempio dell'operaio standard in periodo di alti salari, del burocrate amorfo e ben pagato, ecc. Ed è proprio quest'ultimo genere di esistenza che il socialismo reclama per tutti» ${ }^{82}$. Era, ancora una volta, il philosophe-paysan ad osservare come "[i] democratici moderni hanno troppo frettolosamente confuso vita dura e vita inumana. E con ciò si sono condannati quasi unicamente a corrompere sotto il pretesto di umanizzare» 83 .

Vivere gomito a gomito con altre centinaia d'individui negli squallidi caseggiati di una periferia degradata, d'altronde, può indurre ad un abbrutimento e ad un'incomunicabilità ben maggiori che non il condurre una vita isolata in un modesto casolare di campagna. Potersi sentire, infatti, non significa necessariamente sapersi ascoltare. Aleksandr Soljenicyn rilevava l'abbaglio quasi quarant'anni fa, nel romanzo che gli valse il premio Nobel per la letteratura. Nel tetro clima che caratterizzava il reparto n. 13 (quello oncologico) dell'ospedale sito in una sperduta città sovietica dell'Asia centrale alla fine degli anni cinquanta, l'infermiera Zòja chiedeva al degente Kostoglòtov, il quale le parlava delle tante difficoltà che c'erano nel posto dal quale egli proveniva: «Possibile che si stia così male?»; al che, lui rispondeva: «Niente affatto. È la gente che ha un concetto sbagliato del bene e del male. Si considera bene vivere in una gabbia a cinque piani, con la gente che cammina e batte sopra la testa, e le radio che urlano da tutte le parti. Mentre è considerata una terribile disgrazia la vita laboriosa

82 Thibon (1940: 31, corsivo originale).

83 Ibidem. 
del contadino che abita in una capanna d'argilla al limite della steppa» ${ }^{84}$.

L'alienazione di cui si è trattato in queste pagine, dunque, è quel particolare assoggettamento del lavoro umano alle esigenze peculiari dell'automazione, in base al quale si tende a fare dell'uomo una sorta di accessorio della macchina. Ciò deriva espressamente dalla palese incapacità della tecnica a venire incontro ai bisogni etici, estetici ed affettivi dell'uomo e dalla sua conseguente tendenza a favorire, se non a determinare, l'isolamento degl'individui e la loro reciproca incomunicabilità. Né, d'altronde, può rivelarsi fattore indicativo della bontà di una qualunque azione il semplice fatto che essa venga accettata di buon grado o, perfino, perseguita volontariamente da un soggetto. Una scelta non coercitiva non è anche, per mera definizione, una scelta giusta. Non si devono confondere i distinti piani di valutazione: in veste di filosofo della politica si può legittimamente rivendicare la bontà del principio su cui si fonda la libertà di scelta, rispetto a quello che sostiene l'imposizione coattiva; come storico, sociologo, o antropologo non ci si deve lasciar condizionare dalle proprie preferenze nell'esprimere un giudizio, ma ci si deve sforzare perché questo sia -il più possibile- oggettivo.

Anche chi rivendica la possibilità di drogarsi, ad esempio, in quanto atto - più o meno responsabile-comunque conseguente ad una libera scelta individuale, non può tuttavia negare - a meno che non intenda palesemente rinunciare all'obiettività- gli effetti nefasti che l'uso prolungato di sostanze stupefacenti produce sull'organismo umano. Non si può replicare, al medico che nella sua diagnosi attribuisca all'utilizzo di droghe l'insorgenza nel paziente di un 'sarcoma di Kaposi', di fermarsi a riflettere sul fatto che quella persona ha deciso in piena autonomia di drogarsi poiché da ciò ricavava una

84 Soljenicyn (1967: 44, corsivo aggiunto). 
sensazione di piacere fisico ${ }^{85}$. Non lo si può fare, semplicemente, perché questo non cambierebbe di una virgola la valutazione espressa dal medico. Le due considerazioni, infatti, si collocano su piani paralleli e non comunicanti fra loro. La libertà nella scelta di drogarsi, insomma, non è in grado di neutralizzare gli effetti negativi che tale atto comporta, al pari di quanto avviene per il contadino che si fa operaio volontariamente per faticare di meno e finisce per rimanerne vittima alienandosi.

L'interpretazione più convincente del concetto di alienazione, tuttavia, non appare affatto - nella personale ottica di chi scrive - quella delineatasi all'interno del filone hegelo-marxista, dove si attribuiva all'Ent'fremdung il significato di una perdita di coscienza dell'operaio nel regime capitalistico dovuta principalmente alla proprietà privata e, di conseguenza, superabile soltanto tramite la soppressione della stessa ${ }^{86}$. ऐ̀, piuttosto, quella filtrata attraverso il grande profeta della decadenza della civiltà occidentale, Oswald Spengler ${ }^{87}$, la visione che ha mostrato un'indubbia capacità di svincolarsi da sofismi ideologici tutti interni al medesimo orizzonte ideale, additando nella macchina la causa ultima della decadenza spirituale che attanaglia l'uomo contemporaneo. Il mondo in cui essa domina risulta, nella diagnosi operata dal filosofo tedesco, una realtà senz'anima, livellatrice, mortificante: un

\footnotetext{
85 Già più sensato sarebbe - eventualmente - chiedere al filosofo di fermarsi a riflettere sulle conseguenze, spesso involontarie, che può produrre la libera azione umana. Gli effetti indesiderati dell'eterogenesi dei fini, infatti, viaggiano a doppio senso.

86 Sembra assolutamente convincente, infatti, l'ipotesi sostenuta dal filosofo Lucio Colletti, secondo la quale «[i]l 'feticismo delle merci', la Versachlichung, la Verdinglichung, di cui parla Marx nel Capitale, nei Grundrisse, nelle Theorien über den Mehrwert, sono assolutamente la stessa cosa dell' 'alienazione' o 'estraniamento' (Entäusserung, Entefremdung), cioè allo scambio o inversione (Umkehrung) fra soggetto e predicato, sostrato particolare e universale logico, del quale Marx tratta nella sua critica giovanile di Hegel»: corrispondenza inedita, riportata in Tambosi (1999: 132).

87 Si veda in particolare Spengler (1931).
} 
universo in cui la quantità ha preso il sopravvento sulla qualità e dove il culto sacro dei valori spirituali è stato rimpiazzato da quello utilitaristico degl'interessi strumentali ${ }^{88}$. Per Spengler, dunque, Marx ha perfettamente ragione quando sostiene che la macchina è 'la più superba' creazione della borghesia; e tuttavia il filosofo di Treviri, "succubo dello schema antichitàmedioevo-èra moderna, non ha notato che soltanto la borghesia di una unica civiltà ha finito col dipendere dalla macchina» ${ }^{89}$.

Poiché la macchina domina la terra, infatti, ogni non-europeo, dal giapponese all'indù, dal russo all'arabo, tenta di appropriarsi del segreto di questa terribile arma - proseguiva l'autore del Tramonto dell'Occidente- «ma interiormente la respinge» ${ }^{90}$. Avveniva così che l'ebreo, come imprenditore ed ingegnere rifuggisse dal costruire macchine, per dedicarsi piuttosto al lato affaristico della loro produzione. Allo stesso modo il russo avrebbe guardato con timore e con odio codesta «tirannia delle ruote, dei fili e delle rotaie» al punto che, se anche un giorno o l'altro egli si fosse dovuto piegare alla necessità, adattandosi al suo utilizzo, l'avrebbe poi rimosso dalla propria memoria e dal proprio ambiente "per costruirsi un mondo nel quale nulla più esista di questa tecnica diabolica» ${ }^{91}$.

Realizzare un'economia di mercato creativa, in grado di neutralizzare gli effetti alienanti che sono presenti nel sistema utilitaristico affermatosi in età moderna in seguito alle varie 'rivoluzioni', è — probabilmente- la principale sfida che si pone oggi allo scienziato sociale. Alcuni dei meccanismi tipicamente 'industriali' illustrati in queste pagine risultano, ormai, ampiamente superati. Ma l'essenza del problema sembra ben lungi dal potersi considerare risolta nella sua complessità. La

88 Sul tema mantengono una loro attualità talune considerazioni svolte queste, sì, sulla scia del pensiero hegeliano — da Weil (1934: 108-127).

89 Spengler (1923: 1492, nota 93, corsivo originale).

90 Ibidem.

91 Ibidem (corsivo aggiunto). 
struttura portante del sistema capitalistico, di per sé valida e indiscussa, necessita di riappropriarsi di quella bussola imprescindibile che hanno a lungo rappresentato i valori della morale cristiana. Essi non interferivano in alcun modo con le leggi economiche, stricto sensu, che regolano il libero mercato ma influivano in maniera esclusiva sulla coscienza di ogni singolo attore, condizionando così unicamente il 'foro interno'. Autoconsapevolezza, controllo e competizione appaiono, dunque, come la triade fondante di un'economia di mercato sana, mirata alla costruzione di un modello in grado di soddisfare il bisogno di ricchezza dell'individuo, irrelato agli equilibrî etico-politici della società in cui egli stesso vive.

\section{RIFERIMENTI BIBLIOGRAFICI}

AlbizZeschi, Bernardino (1963), Sermones XXXII-XLV. De contractibus et usuris, in Bernardinus Senensis, Sermones imperfecti, Itinerarium anni, Epistolae, edd. PP. Collegii S. Bonaventurae, Firenze, Quaracchi-Collegio S. Bonaventura, vol. VIII di S. Bernardini Senensis opera omnia.

Ambrosetti, G. (1973), Diritto privato ed economia nella Seconda Scolastica, in Paolo Grossi (a cura di), La seconda Scolastica nella formazione del diritto privato moderno, Milano, Dott. A. Giuffrè Editore.

ANDREAU, Jean (1987), Vie financière dans le monde romain, Les métiers de manieurs d'argent (IVe siècle av. J.-C.-III siècle ap. J.-C.), Roma, École Française de Rome.

- (1999), Banking and Business in the Roman World, 310 B.C.A.D. 284, Cambridge and New York, Cambridge University Press.

Aquino, Tommaso di (1266-73), Summa Theologiae, fino alla parte III, questione 90 inclusa, di cui la I parte, 1266-68; la I-II, 1269-71; la II-II, 1271-72; la III, 1272-73 [il resto, ossia il Supplemento, è tratto dal Commento alle Sentenze]. 
Bentham, Jeremy (1787), Defence of Usury, London, 1790.

BLock, Walter (1976), Defending the Undefendable: The Pimp, Prostitute, Scab, Slumlord, Libeler, Moneylender, and Other Scapegoats in the Rogue's Gallery of American Society, San Francisco, Fox \& Wilkes, 1991, trad. it. Difendere l'indifendibile, Macerata, Liberilibri, 1993.

CAstiglioni, Luigi y MARIOTti, Scevola (1966), Il vocabolario della lingua latina, redatto con la collaborazione di A. Brambilla e G. Campagna, Torino, Loescher Editore.

Cipolla, Carlo M. (1974), Storia economica dell'Europa preindustriale, Bologna, Società editrice il Mulino, 2003.

Evola, Julius (1953), Gli uomini e le rovine, Roma, Edizioni Mediterranee, 1998.

FinZI, Roberto (1987), Corso di Storia (3 voll.), Bologna, Nicola Zanichelli Editore, 1990, vol. I: L'età medievale.

GuÉnon, René (1945), Le Règne de la Quantité et les Signes des Temps, Paris, Éditions Gallimard, trad. it. Il Regno della Quantità e i Segni dei Tempi, Milano, Adelphi Edizioni, 1982.

Huerta de Soto, Jesús (1998), Dinero, crédito bancario y ciclos económicos, Madrid, Unión Editorial, 2002.

- (2001), La Escuela Austriaca: mercado y creatividad empresarial, Madrid, Editorial Síntesis, trad. it. La Scuola Austriaca. Mercato e creatività imprenditoriale, a cura di Paolo Zanotto, prefazione di Raimondo Cubeddu, Soveria Mannelli (CZ), Rubbettino Editore, 2003.

JASPERS, Karl (1925), Psychologie der Weltanschauungen, Berlin, Verlag von Julius Springer, trad. it. Psicologia delle visioni del mondo, Roma, Casa Editrice Astrolabio-Ubaldini Editore, 1950.

Mandeville, Bernard de (1714), The fable of the bees: or, private vices, publick benefits, London, 1723, trad. it. La favola delle api: ovvero vizi privati, pubblici benefici con un saggio sulla carità e le scuole di carità e un'indagine sulla natura della 
società, a cura di Tito Magri, Roma-Bari, Giuseppe Laterza \& Figli Editori, 2002.

- (1724), A modest defence of publick stews: or, an essay upon whoring, as it is now practis'd in these kingdoms, London, trad. it. Una modesta difesa delle case di piacere, a cura di Giacinto Borelli, Soveria Mannelli (CZ), Rubbettino Editore, 1995.

Nelson, Benjamin (1967), Usura e cristianesimo. Per una storia della genesi dell'etica moderna, Firenze, G. C. Sansoni editore.

Pierozzi, Antonino (1440-59), Summa theologiae moralis, Venezia, Joannes de Colonia, 1477.

Polanyi, Karl (1944), The Great Transformation: The Political and Economic Origins of Our Time, New York, Holt, Rinehart \& Winston Inc., trad. it. La grande trasformazione. Le origini economiche e politiche della nostra epoca, Torino, Giulio Einaudi editore, 1974.

Ricciotтi, Giuseppe [a cura di] (1940), La Sacra Bibbia, Firenze, Casa Editrice Adriano Salani.

Rothbard, Murray N. (1982), The Ethics of Liberty, New York and London, New York University Press, 1998.

- (1995), An Austrian Perspective on the History of Economic Thought (2 voll.), vol. I: Economic Thought before Adam Smith, vol. II: Classical Economics, Cheltenham (UK) and Brookfield (VT), Edward Elgar Publishing.

SANTARELLI, Umberto (1984), La categoria dei contratti irregolari. Lezioni di Storia del diritto, Torino, G. Giappichelli Ed, 1998.

- (1989) Mercanti e società tra mercanti, Torino, G. Giappichelli Editore, 1992.

Schumpeter, Joseph A. (1954), History of Economic Analysis, edited from manuscript by Elizabeth Boody Schumpeter, Oxford and New York, Oxford University Press.

SolJENicyn, Aleksandr I. (1967), Ràkovyi kòrpus, trad. it. Padiglione cancro, Roma, Biblioteca Economica Newton (Classici BEN), 1994. 
SOMBART, Werner (1913), Der Bourgeois. Zur Geistsgeschichte des Modernen Wirtschaftsmenschen, München und Leipzig, Duncker \& Humblot, trad. it. Il borghese. Lo sviluppo e le fonti dello spirito capitalistico, Traduzione di Henry Furst, Presentazione di Franco Ferrarotti, Parma, Ugo Guanda Editore, 1994.

SPENGLer, Oswald (1923), Der Untergang des Abendlandes. Umrisse einer Morphologie der Weltgeschichte, Vollständige Ausgabe in einem Band, München, C. H. Beck'sche Verlagsbuchhandlung (Oscar Beck), trad. it. Il tramonto dell'Occidente. Lineamenti di una morfologia della Storia mondiale, Parma, Ugo Guanda Editore, 2002.

- (1931), Der Mensch und die Technik. Beitrag zu einer Philosophie des Lebens, München, C. H. Beck'sche Verlagsbuchhandlung (Oscar Beck), trad. it. L'uomo e la tecnica. Contributo a una filosofia della vita, Parma, Ugo Guanda Editore, 1992.

SPICCIANI, Amleto (1990), Capitale e interesse tra mercatura e povertà nei teologi e canonisti dei secoli XIII-XV, Roma, Jouvence.

TAmbosi, Orlando (1999), O declínio do marxismo e a herança hegeliana (L. Colletti e o debate italiano: 1945-1991), Florianópolis, Editora da Universidade Federal de Santa Catarina, trad. it. Perché il marxismo ha fallito. Lucio Colletti e la storia di una grande illusione, Milano, Arnoldo Mondadori Editore, 2001.

Thibon, Gustave (1940), Diagnostics. Essai de physiologie sociale, Paris, Librairie de Médicis, 1945, trad. it. in Id., Ritorno al reale. Prime e seconde diagnosi in tema di fisiologia sociale, Prefazione di Gabriel Marcel, a cura e con considerazioni introduttive di Marco Respinti, Milano, Effedieffe Ed., 1998.

Tosato, Angelo (2002), Vangelo e ricchezza. Nuove prospettive esegetiche, a cura di Dario Antiseri, Francesco D'Agostino e Angelo Petroni, Soveria Mannelli (CZ), Rubbettino Editore. 
Vito, Francesco (1967), Introduzione alla economia politica, Milano, Dott. A. Giuffrè Editore.

WeIL, Simone (1934), Réflexions sur les causes de la liberté et de l'oppression sociale, Paris, Éditions Gallimard, 1955, trad. it. Riflessioni sulle cause della libertà e dell'oppressione sociale, Milano, Adelphi Edizioni, 1997. 